\title{
Strategic Incision Placement to Facilitate Successful Supermicrosurgical Lymphaticovenular Anastomoses
}

\author{
Patrick J Hawkes, MD; Maria McNurlen; Mindy Bowen; Wei F. Chen, MD, FACS* \\ Division of Plastic and Reconstructive Surgery, Department of Surgery, \\ University of lowa Hospitals and Clinics, lowa City, lowa, USA
}

\begin{abstract}
Introduction: Supermicrosurgical lymphaticovenular anastomosis (LVA) has become an accepted and effective treatment for lymphedema. Surgeons performing these procedures, however, have been perplexed in determining where to place their incisions to maximize the number of LVAs constructed. Here, we describe our guided approach to incision placement to increase the likelihood of creating a successful LVA at each incision. Methods: Thirteen consecutive patients underwent LVA for treatment of secondary lymphedema. Incisions were placed using the guided approach in all the patients. Additional incisions were placed using the blind (anatomic) approach when the appropriate number of LVAs had not been achieved. In the guided approach, superficial lymphatics were mapped intraoperatively with indocyanine green (ICG) lymphography and superficial venules were mapped intraoperatively with near-infrared (NIR) vein visualization (Figure 2). Guided incisions were then placed where lymphatic vessels and superficial venules came into close contact or intersected (Figure 3). In the blind approach, incisions were placed along the anatomic course of the cephalic or greater saphenous vein (Figure 3). The number of LVAs constructed using each approach was compared. Fisher's exact test was used for statistical analysis.

Results: A total of 99 LVAs were created through 80 incisions. Twelve of 31 (39\% success) incisions using the blind approach and 42 of 49 ( $86 \%$ success) incisions using the guided approach allowed for successful LVA construction. The guided approach also resulted in more LVAs created per incision (1.7 vs. 0.5, $\mathrm{P}=0.0001$ ).

Conclusion: Use of a multimodality image guided approach significantly increases the probability of successful LVA creation at each incision as well as the total number of LVAs that are created within each incision.
\end{abstract}

\section{Introduction}

Lymphedema is a chronic and progressive disease that has been historically difficult to manage. Cancer treatments, especially those involving lymph node dissections, have contributed to the increasing prevalence of the disease. Supermicrosurgical lymphaticovenular anastomosis (LVA) has been shown to effectively treat lymphedema [1-4]. In LVA, lymphatic vessels are connected to veins through a series of small skin incisions. The anastomoses create additional outflow conduits to improve lymphatic drainage.

Successful construction of an LVA requires identification of both adequate caliber lymphatic vessels and venules within each incision site. Lymphatic vessels used in LVA must be patent and located near veins of compatible size. If the vein is too large, pressure in the lymphatic vessel will be insufficient to overcome the venous blood flow and the LVA will not function. If the vein is too small, the anastomosis may be technically difficult to complete (currently $0.2 \mathrm{~mm}$ is the smallest vessel size used at our institution). One of the difficulties the surgeons encounter while performing LVA is in determining where to place incisions to access veins and lymphatic vessels meeting these criteria.

LVA incisions were historically placed in a blind fashion. Surgeons mapped superficial veins using anatomic landmarks, such as the cephalic vein in the upper extremity and the greater saphenous vein in the lower extremity [4]. To increase the likelihood of encountering lymphatic vessels, LVA incisions were typically clustered distally where tissues were thinner and vessels were present at a higher concentration [1,2]. To improve the success rate of encountering adequate lymphatics and veins at LVA incision sites, surgeons began using intraoperative ICG lymphography guidance $[5,6]$. Although ICG lymphography allowed the surgeons to encounter lymphatic vessels with higher frequency, it did not improve the probability of finding the nearby venules. This led to combining ICG lymphography and near-infrared (NIR) vein visualization, which had resulted in successful outcomes $[7,8]$. However, there have not been any studies to our knowledge directly comparing the success rates of this mapped approach and the traditional blind (anatomical) mapping. Here, we describe a method of guided incision placement and compare the rate of successful LVA formation to that found in the blind approach.

\section{Methods}

\section{Patient Selection and Evaluation}

Following IRB approval, the patients at the University of lowa Hospitals and Clinics who underwent LVA for treatment of secondary lymphedema between July 2015 and December 2015 were recruited for study [9,10]. Disease severity was staged clinically using Campisi criteria (Table 1) and radiographically using indocyanine green (ICG) lymphography staging criteria previously described by Yamamoto et al [11]. Patient assessment was performed preoperatively and at three and six months postoperatively. The assessment included patient-reported relief of symptoms, clinical exam, validated lymphedema quality of life assessment, and ICG lymphography [12].

\section{Vessel Mapping}

In the guided approach, superficial lymphatics were mapped intraoperatively with ICG lymphography by injecting $0.1 \mathrm{~mL}$ of $0.25 \%$ ICG intradermally at the first and second web spaces of the foot or the second and third web spaces of the hand (Figure 1A). The injected limb was scanned with the SPY Elite system (Life-Cell Corp., Bridgewaterer, NJ) immediately following injections to visualize the superficial lymphatic vessels. Lymphatic vessels were marked with a solid line based on injection site (Figure 1B). Additional injections were performed until no drainage from the most recent injection was visualized. 
ORIGINAL

Table 1. Staging Criteria for Lymphedema

\begin{tabular}{|c|c|c|c|c|c|}
\hline \multicolumn{2}{|c|}{ Stage I } & \multirow{2}{*}{ Stage II } & \multirow{2}{*}{ Stage III } & \multirow{2}{*}{ Stage IV } & \multirow{2}{*}{ Stage V } \\
\hline A & B & & & & \\
\hline $\begin{array}{l}\text { No overt swelling } \\
\text { despite impaired } \\
\text { lymph drainage }\end{array}$ & $\begin{array}{l}\text { Reversible swelling } \\
\text { subsiding with limb } \\
\text { elevation }\end{array}$ & $\begin{array}{l}\text { Swelling that only } \\
\text { partially reduces with } \\
\text { limb elevation }\end{array}$ & $\begin{array}{l}\text { Persistent edema } \\
\text { with recurrent } \\
\text { lymphangitis }\end{array}$ & $\begin{array}{l}\text { Fibrotic lymphedema } \\
\text { with column-limb }\end{array}$ & $\begin{array}{l}\text { Progression to } \\
\text { elephantiasis with } \\
\text { limb deformation } \\
\text { including widespread } \\
\text { lymphostatic warts }\end{array}$ \\
\hline
\end{tabular}

Staging criteria for lymphedema is adapted from Campisi, et al. [5,6].

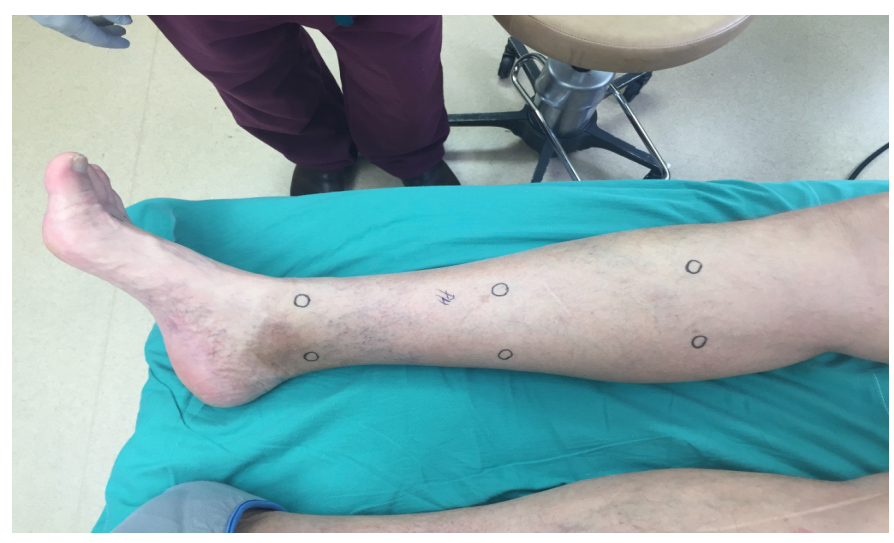

A

Figure 1. A, marked sites for sequential distal-to-proximal ICG injection. B, lymphatic vessels visualized using ICG lymphography marked with solid lines in colors corresponding to injection site.

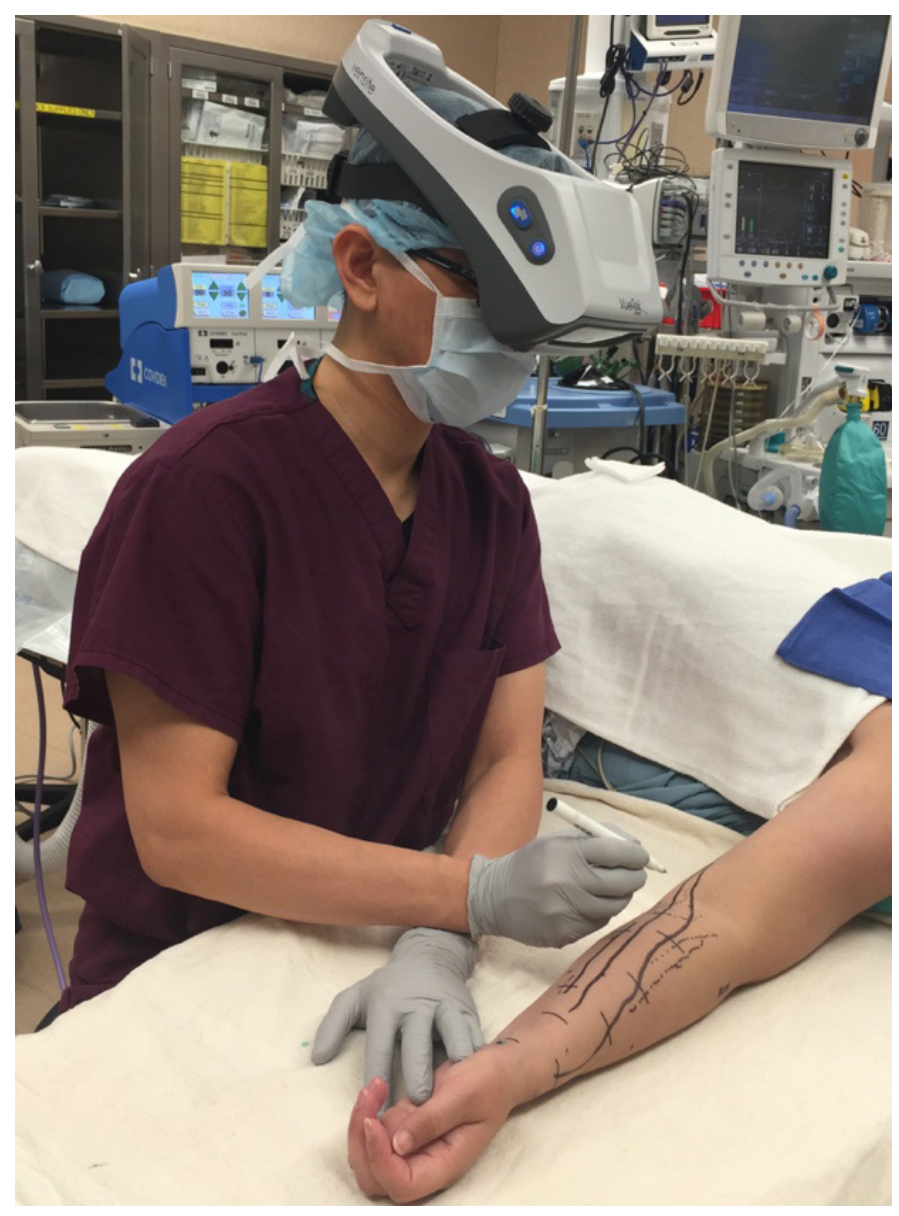

A

Figure 2. A, marking the superficial veins of upper extremity with the Veinsite (VueTek Scientific ${ }^{\circledR}$, Gray, ME). B, vein marking with marking pen as viewed through the Veinsite. C, upper extremity with veins (dotted lines) marked.

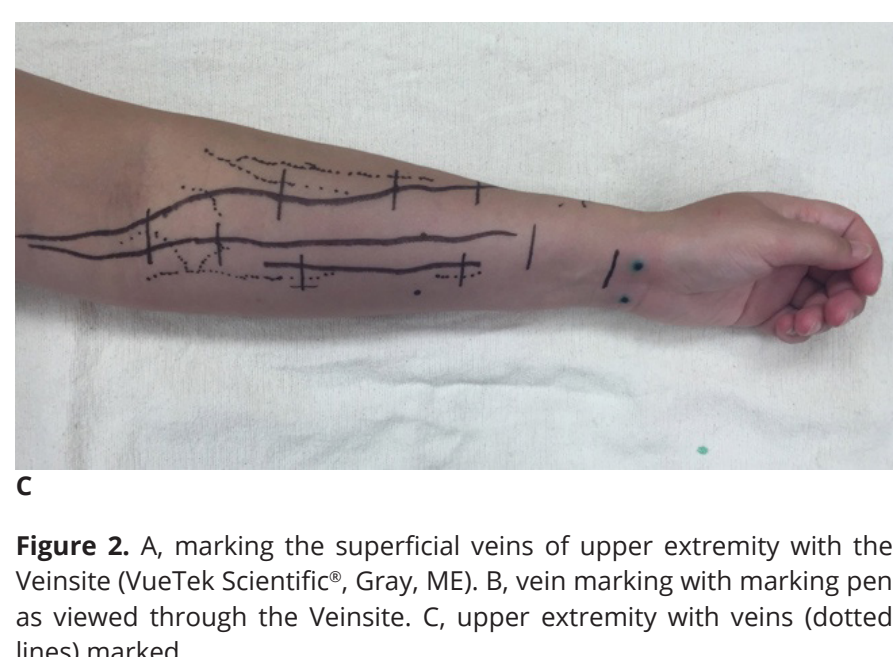



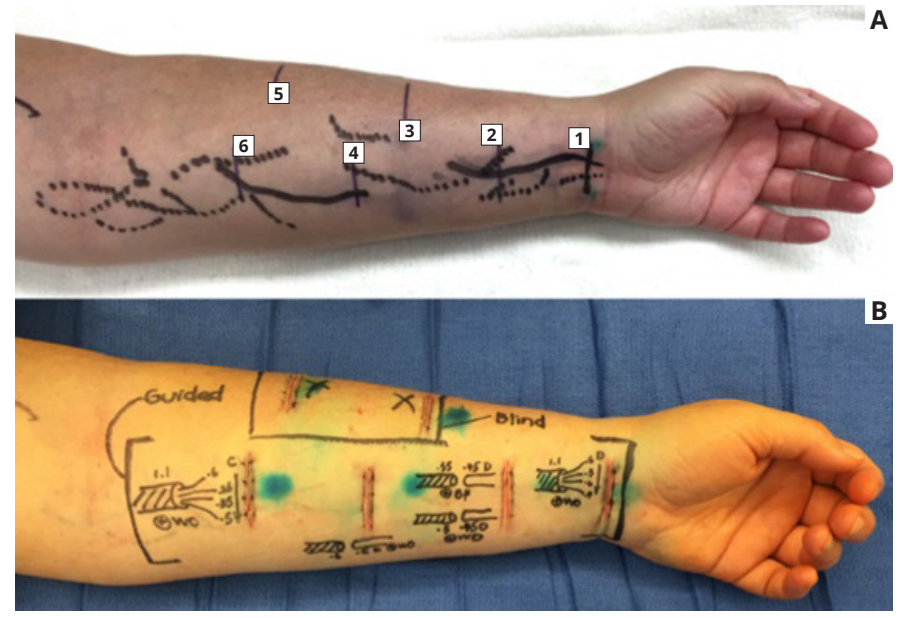

Figure 3. A, guided incisions (1, 2, 4, and 6 on volar forearm) marked at sites of intersecting or nearby veins (dotted lines) and lymphatic vessels (solid lines). Blind incisions ( 3 and 5 on radial forearm) marked along the course of the cephalic vein. B, postoperative demonstration showing successful LVA construction at guided incision sites and unsuccessful LVA construction at blind incision sites.

Superficial venules were mapped using the Veinsite (VueTek Scientific ${ }^{\circledR}$, Gray, ME) near-infrared (NIR) vein visualization and marked with a dotted line (Figure 2C, 3A). Incision sites for LVA were marked where solid and dotted lines intersected or were in close proximity.

\section{Operative Technique}

Following incision markings, $0.1 \mathrm{~mL}$ of $1 \%$ Lymphazurin was injected approximately $2 \mathrm{~cm}$ distal to each incision to aid in intraoperative visualization of the lymphatic vessels. Dissection was performed through 3 $\mathrm{cm}$ incisions under $18 \mathrm{x}$ to $22 \mathrm{x}$ magnification with a surgical microscope
(Pentero 900; Carl Zeiss, Oberkochen, Germany). Supermicrosurgical instruments (EMI Factory, Kitasakugun, Japan) were used for vessel dissection. Anastomoses were performed using 11-0 and 12-0 Nylon sutures (Crown Jun, 50 $\mu$ needle, Ichikawashi, Chiba, Japan) using techniques previously described [13-15].

The blind approach to incision placement was utilized when fewer than 10 LVAs were created with the guided approach or when the quality of the lymphatic vessels was insufficient (Table 2). Lymphatic vessel quality was determined visually and categorized as good (normal or ectatic), suboptimal (contracted), or unusable (sclerotic) [16]. When indicated, blind incisions were made following the anatomic course of the cephalic or greater saphenous vein (Figure 3A). Operative times for each case were obtained from the case log and vessel mapping times were recorded. Fisher's exact test was used for statistical analysis.

\section{Results}

\section{Patient Selection and Evaluation}

Thirteen patients with lymphedema Campisi stage Ib to III and lymphographic stage II to V met the inclusion criteria (Table 2). All the thirteen patients had uneventful postoperative courses and were discharged one day postoperatively. The follow-up period ranged from five to nine months; no patients were lost to follow up. At their follow-up appointments, all patients reported a decrease in lymphedema-related symptoms that paralleled their improved findings on clinical exam, validated quality of life assessment, and ICG lymphography.

\section{LVA Completion}

A total of 99 LVAs were created through 80 incisions by senior author WFC. Forty-two of 49 ( $86 \%$ success) incisions using the guided approach resulted in successful completion of LVA (Table 2). Twelve of 31 (39\% success) incisions using the blind approach resulted in successful completion of LVA (Table 2). The guided approach allowed construction of 1.7 LVAs per incision, while the blind approach allowed construction of 0.5 LVAs per incision (Table 2). The average operative time for the thirteen patients was $4.8 \pm 0.5$ hours and the time spent for mapping vessels was $13 \pm 3$ minutes.

Table 2. Characteristics of Patients Undergoing LVA and Surgical Outcomes

\begin{tabular}{|c|c|c|c|c|c|c|c|c|c|c|c|c|}
\hline \multirow[t]{2}{*}{ Patient } & \multirow[t]{2}{*}{ Age } & \multirow[t]{2}{*}{ Sex } & \multirow[t]{2}{*}{ Extremity } & \multicolumn{2}{|c|}{ Stage } & \multicolumn{3}{|c|}{ Guided Incision Placement } & \multicolumn{3}{|c|}{ Blind Incision Placement } & \multirow[t]{2}{*}{$P$} \\
\hline & & & & Clinical & Lymph & $\begin{array}{l}\text { Total } \\
\text { Incisions }\end{array}$ & $\begin{array}{l}\text { Successful } \\
\text { Incisions }\end{array}$ & $\begin{array}{l}\text { LVAs } \\
\text { Created }\end{array}$ & $\begin{array}{l}\text { Total } \\
\text { incisions }\end{array}$ & $\begin{array}{l}\text { Successful } \\
\text { incisions }\end{array}$ & $\begin{array}{l}\text { LVAs } \\
\text { created }\end{array}$ & \\
\hline 1 & 43 & $\mathrm{~F}$ & L arm & ॥ & IV & 5 & 5 & 11 & 0 & 0 & 0 & \\
\hline 2 & 52 & $\mathrm{~F}$ & L arm & II & III & 4 & 4 & 11 & 2 & 0 & 0 & \\
\hline 3 & 50 & $\mathrm{~F}$ & L leg & II & III & 6 & 5 & 6 & 1 & 0 & 0 & \\
\hline 4 & 66 & $\mathrm{~F}$ & $\mathrm{R}$ arm & III & IV & 2 & 2 & 2 & 3 & 1 & 1 & \\
\hline 5 & 42 & $\mathrm{~F}$ & $\mathrm{~L}$ arm & III & IV & 2 & 2 & 7 & 4 & 2 & 4 & \\
\hline 6 & 61 & $\mathrm{~F}$ & $\mathrm{R}$ arm & III & III & 4 & 4 & 11 & 0 & 0 & 0 & \\
\hline 7 & 44 & $M$ & $\mathrm{R}$ arm & III & IV & 0 & 0 & 0 & 7 & 3 & 4 & \\
\hline 8 & 70 & $\mathrm{~F}$ & L arm & III & IV & 5 & 4 & 4 & 1 & 0 & 0 & \\
\hline 9 & 48 & $\mathrm{~F}$ & R leg & II & III & 6 & 5 & 8 & 1 & 0 & 0 & \\
\hline 10 & 29 & $\mathrm{~F}$ & L leg & II & V & 0 & 0 & 0 & 6 & 3 & 3 & \\
\hline 11 & 65 & $\mathrm{~F}$ & L leg & III & III & 6 & 4 & 5 & 2 & 1 & 1 & \\
\hline 12 & 62 & $\mathrm{~F}$ & $\mathrm{~L}$ arm & II & III & 5 & 4 & 11 & 2 & 1 & 2 & \\
\hline \multirow[t]{4}{*}{13} & 51 & $\mathrm{~F}$ & $\mathrm{R}$ arm & IB & III & 4 & 3 & 6 & 2 & 1 & 2 & \\
\hline & & & & & Total: & 49 & 42 & 82 & 31 & 12 & 17 & \\
\hline & & & & & \multicolumn{3}{|c|}{ Success rate } & $85.7 \%$ & & & $38.7 \%$ & 0.0001 \\
\hline & & & & & \multicolumn{3}{|c|}{ LVAs per incision } & 1.5 & & & 0.5 & 0.0001 \\
\hline
\end{tabular}

Abbreviations: LVA, lymphaticovenous anastomosis; Lymph, lymphangiographic. 


\section{Discussion}

The goal of surgical treatment of lymphedema with LVA is to provide alternative drainage routes for excessive accumulation of lymph. To successfully construct an LVA, the surgeon must identify both suitable lymphatic vessel (normal, ectactic, or contracted) and suitable vein. Both the lymphatic vessel and vein must be of compatible size and close enough to be connected.

In this patient population, utilization of guided incision placement with multi-modality intraoperative imaging increased successful LVA construction at each incision $(86 \%$ vs. $39 \%, P=0.0001)$ and also increased the number of LVAs constructed within each incision (1.7 vs. $0.5, P=0.0001$ ). Although mapping with ICG lymphography and NIR added 10-15 minutes to the operative time, the overall operative time decreased when this technique was used. We attributed this to the ability to complete more successful LVAs using fewer incisions. While lymphatic and venous mapping equipment must be purchased to perform the guided incision approach, this is a one-time fixed cost. Thereafter, performing venous mapping has no additional expense. Performing ICG lymphography requires ICG dye and an injection syringe with needle, neither of which adds appreciable cost to the LVA procedure.

We acknowledge that by using the blind approach only when the lymphatic vessels were inadequate in quality or LVAs were inadequate in number, a degree of selection bias was present in our study design. The study design was selected because by using the guided incision placement in every patient when able, we could create more LVAs and optimize the individual patient outcomes following surgery.

While it has been demonstrated that the patient outcomes are improved when more LVAs are made $[17,18]$, the optimal number of LVAs is unknown. The goal is to maximize lymphatic drainage pathways. We recommend surgeons who are skilled in various anastomotic configurations. Too often, surgeons rely on end-to-end anastomoses, which create fewer drainage pathways than other configurations. Currently at our institution, we determine surgical endpoint based on quality and quantity of LVAs constructed, patient anesthesia time, and surgeon fatigue. The average operative time of the patients in this study ( 4.8 hours \pm 0.5 hours) was based on this approach. In future studies, we hope to explore criteria for a more definitive LVA operation endpoint.

Many surgeons who perform the LVA consider the absence of linear pattern finding on ICG lymphography to be a contraindication to the procedure. This study demonstrated that even in the absence of linear pattern ICG lymphographic findings, we still identified suitable lymphatic vessels and created successful LVAs, albeit at a lower success rate (Table 2). Based on these findings, we do not consider the presence of a linear ICG lymphographic pattern to be a prerequisite for successful surgery. We prefer a guided approach for LVA when possible but still offer the procedure in patients without linear patterns on ICG lymphography.

\section{Conclusion}

Combining intraoperative ICG lymphography with NIR vein visualization can aid supermicrosurgeons in identifying lymphatic vessels and superficial venules to guide LVA incision placement. This guided approach significantly improves successful creation of LVAs when compared to the blind (anatomic) approach. In addition, the absence of linear ICG lymphographic patterns does not prevent formation of successful LVAs.

\section{Article Information}

\section{*Correspondence: Wei F. Chen, MD, FACS}

Division of Plastic and Reconstructive Surgery, Department of Surgery, University of lowa Hospitals and Clinics, 20 Hawkins Drive, 1537 JCP, lowa City, lowa 52242, USA. Email: wei-chen@uiowa.edu
Received: May. 12, 2017; Accepted: Oct. 18, 2017; Published: Jan. 2, 2018

\section{DOI: 10.24983/scitemed.imj.2018.00049}

Copyright (c) 2018 The Author(s). This is an open-access article distributed under the terms of the Creative Commons Attribution 4.0 International License (CC-BY).

\section{Funding: None}

\section{Conflict of Interest Disclosures: None}

\section{Keywords}

Lymphedema; lymphaticovenular anastomosis; supermicrosurgery.

\section{References}

1. Yamamoto T, Narushima M, Yoshimatsu H, et al. Minimally invasive lymphatic supermicrosurgery (MILS): indocyanine green lymphography-guided simultaneous multisite lymphaticovenular anastomoses via millimeter skin incisions. Annals of Plastic Surgery 2014;72:6770.

2. Koshima I, Inagawa K, Etoh K, et al. Supramicrosurgical lymphaticovenular anastomosis for the treatment of lymphedema in the extremities. Journal of Reconstructive Microsurgery 2000;100:437-442.

3. Campisi $C$, Davini D, Bellini C, et al. Lymphatic microsurgery for the treatment of lymphedema. Microsurgery 2006;26:65-69.

4. Seki Y, Yamamoto T, Yoshimatsu H, et al. The superior-edge-ofthe-knee incision method in lymphaticovenular anastomosis for lower extremity lymphedema. Plastic and Reconstructive Surgery 2015;136:665e-675e.

5. Mihara M, Seki $Y$, Hara $H$, et al. Predictive lymphatic mapping: a method for mapping lymphatic channels in patients with advanced unilateral lymphedema using indocyanine green lymphography. Annals of Plastic Surgery 2014;72:706-710.

6. Liu HL, Pang SY, Chan YW. The use of a microscope with near-infrared imaging function in indocyanine green lymphography and lymphaticovenous anastomosis. Journal of Plastic, Reconstructive \& Aesthetic Surgery 2014;67:231-236.

7. Mihara M, Hara H, Hayashi $\mathrm{Y}$, et al. Upper-limb lymphedema treated aesthetically with lymphaticovenous anastomosis using indocyanine green lymphography and noncontact vein visualization. Journal of Reconstructive Microsurgery 2012;28:327-332.

8. Mihara M, Hara $H$, Kikuchi $K$, et al. Scarless lymphatic venous anastomosis for latent and early-stage lymphoedema using indocyanine green lymphography and non-invasive instruments for visualising subcutaneous vein. Journal of Reconstructive Microsurgery 2012;65:1551-1558.

9. Campisi C, Campisi C, Accogli S, Campisi C, Boccardo F. Lymphedema staging and surgical indications in geriatric age. BMC Geriatrics 2010;10(Suppl 1):A50.

10. Campisi C, Boccardo F. Lymphedema and microsurgery. Microsurgery 2002;22:74-80.

11. Yamamoto T, Yamamoto N, Doi K, et al. Indocyanine green (ICG)-enhanced lymphography for upper extremity lymphedema: a novel severity staging system using dermal backflow (DB) patterns. Plastic and Reconstructive Surgery 2011;128:941-947.

12. Pyszel A, Malyszczak K, Pyszel K, et al. Disability, psychological distress and quality of life in breast cancer survivors with arm lymphedema. Lymphology 2006;39:185-192.

13. Lasso JM, Perez Cano R. Practical solutions for lymphaticovenous anastomosis. Journal of Reconstructive Microsurgery 2013;29:1-4.

14. Yamamoto T, Yoshimatsu H, Narushima M, et al. A modified side-toend lymphaticovenular anastomosis. Microsurgery 2013;33:130-133.

15. Chen WF, Yamamoto T, Fisher M, et al. The "Octopus" lymphaticovenular anastomosis: evolving beyond the standard super- 


\section{ORIGINAL}

microsurgical technique. Journal of Reconstructive Microsurgery 2015;31:450-457.

16. Narushima M, Yamamoto T, Ogata F, et al. Indocyanine green lymphography findings in limb lymphedema. Journal of Reconstructive Microsurgery 2016;32:72-79.
17. Kung TA, Champaneria MC, Maki JH, Neligan PC. Current concepts in the surgical management of lymphedema. Plastic and Reconstructive Surgery 2017;139:1003e-1013e.

18. Obrien BM, Sykes P, Threlfall GN, Browning FS. Microlymphaticovenous anastomoses for obstructive lymphedema. Plastic and Reconstructive Surgery 1977;60:197-211. 